\title{
Nuclear energy density functional from chiral pion-nucleon dynamics revisited 1
}

\author{
N. Kaiser and W. Weise \\ Physik Department, Technische Universität München, D-85747 Garching, Germany \\ email:nkaiser@ph.tum.de
}

\begin{abstract}
We use a recently improved density-matrix expansion to calculate the nuclear energy density functional in the framework of in-medium chiral perturbation theory. Our calculation treats systematically the effects from $1 \pi$-exchange, iterated $1 \pi$-exchange, and irreducible $2 \pi$-exchange with intermediate $\Delta$-isobar excitations, including Pauli-blocking corrections up to three-loop order. We find that the effective nucleon mass $M^{*}(\rho)$ entering the energy density functional is identical to the one of Fermi-liquid theory when employing the improved density-matrix expansion. The strength $F_{\nabla}(\rho)$ of the $(\vec{\nabla} \rho)^{2}$ surface-term as provided by the pion-exchange dynamics is in good agreement with that of phenomenological Skyrme forces in the density region $\rho_{0} / 2<\rho<\rho_{0}$. The spin-orbit coupling strength $F_{\text {so }}(\rho)$ receives contributions from iterated $1 \pi$-exchange (of the "wrong sign") and from three-nucleon interactions mediated by $2 \pi$-exchange with virtual $\Delta$-excitation (of the "correct sign"). In the region around $\rho_{0} / 2 \simeq 0.08 \mathrm{fm}^{-3}$ where the spin-orbit interaction in nuclei gains most of its weight these two components tend to cancel, thus leaving all room for the short-range spin-orbit interaction. The strength function $F_{J}(\rho)$ multiplying the square of the spin-orbit density comes out much larger than in phenomenological Skyrme forces and it has a pronounced density dependence.
\end{abstract}

PACS: 12.38.Bx, 21.30.Fe, 21.60.-n, 31.15.Ew

Keywords: Nuclear energy density functional; Density-matrix expansion; Chiral pion-nucleon dynamics

\section{Introduction}

The nuclear energy density functional approach is the many-body method of choice in order to calculate the properties of medium-mass and heavy nuclei in a systematic manner [1]. In this context non-relativistic Skyrme forces [2, 3, 4, 5, 6] have gained much popularity because of their analytical simplicity and their ability to reproduce nuclear properties over the whole periodic table within the self-consistent Hartree-Fock approximation. Another widely and successfully used approach to nuclear structure calculations are relativistic mean-field models [7, 8]. In these models the nucleus is described as a collection of independent Dirac quasi-particles moving in self-consistently generated scalar and vector mean-fields. The footprints of relativity become visible through the large nuclear spin-orbit interaction which emerges in that framework from the interplay of the strong scalar and vector mean-fields. These counteract in producing the (attractive) central potential but act coherently to generate the strong spin-orbit potential.

\footnotetext{
${ }^{1}$ Work supported in part by BMBF, GSI and the DFG cluster of excellence: Origin and Structure of the Universe.
} 
Constraints from chiral (pion-nucleon) dynamics and the symmetry breaking pattern of QCD at low energies have been implemented into a pertinent relativistic point-coupling Lagrangian in ref. [9]. In such a more constrained scheme the results for nuclear observables come out comparable to those of the best phenomenological parameterizations of relativistic mean-field models.

A complementary approach in the quest for predictive nuclear energy density functionals [10, 11, 12 focusses less on the fitting of experimental data, but attempts to constrain the analytical form of the functional and the values of its couplings from many-body perturbation theory and the underlying two- and three-nucleon interactions. Switching from the conventional hard-core NN-potentials to low-momentum interactions is essential in this respect, because the nuclear many-body problem formulated in terms of low-momentum interactions becomes significantly more perturbative. Indeed, second-order perturbative calculations provide already a good account of the bulk correlations in infinite nuclear matter [13] and in doubly-magic nuclei [14].

In many-body perturbation theory the contributions to the energy are written in terms of density-matrices and propagators convoluted with the finite-range interaction vertices, and are therefore highly non-local in both space and time. In order to make such functionals numerically tractable in heavy open-shell nuclei it is desirable to develop simplified approximations to these functionals expressed in terms of local densities and currents only. At this stage of the procedure the density-matrix expansion comes prominently into play as it removes the non-local character of the exchange (Fock) contribution to the energy by mapping it onto a generalized Skyrme functional with density dependent couplings. Until recently, the prototype for that has been the density-matrix expansion of Negele and Vautherin [15]. This version of the density-matrix expansion (in particular its Fourier-transform to momentum space) has also been used in refs. [16, 17] to calculate the nuclear energy density functional in the framework of in-medium chiral perturbation theory. The density-dependent coupling strengths of the surface term, $(\vec{\nabla} \rho)^{2}$, or the spin-orbit term, $\vec{\nabla} \rho \cdot \vec{J}$, arise in these calculations exclusively from the longrange $1 \pi$ - and $2 \pi$-exchange dynamics in an inhomogeneous many-nucleon system characterized by a local density $\rho(\vec{r})$ and a local spin-orbit density $\vec{J}(\vec{r})$.

In a recent paper by Gebremariam, Duguet and Bogner [18] an improved density-matrix expansion has been developed for spin-unsaturated nuclei. It has been demonstrated that phase-space averaging techniques allow for a consistent expansion of both the spin-independent (scalar) part as well as the spin-dependent (vector) part of the density-matrix. A further key feature of the new method has been to take into account the deformation displayed by the local density distribution at the surface of most nuclei. The accuracy of the new phase-space averaged density-matrix expansion and the original one of Negele and Vautherin has been gauged via the Fock energy (densities) arising from (schematic finite-range) central, tensor and spin-orbit interactions for a large set of semi-magic nuclei. For a central force the Fock energy depends primarily on the spin-independent (scalar) part of the density-matrix and a few percent accuracy is reached for both variants of the density-matrix expansion. On the other hand the Fock energy due to a tensor force is determined by the spin-dependent (vector) part of the density-matrix. In that case the original density-matrix expansion of Negele and Vautherin leads to an error of about $50 \%$, whereas the new one based on phase-space averaging techniques reduces the error drastically to only a few percent. This is the same level of accuracy as obtained for interaction terms involving the spin-independent (scalar) part of the density-matrix. For further details on these extensive and instructive test studies we refer to ref. [18.

The purpose of the present work is to match with these new developments and to reconsider the nuclear energy density functional as it emerges from chiral pion-nucleon dynamics on the 
basis of the improved density-matrix expansion of ref.[18]. Our paper is organized as follows. In section 2 we recall the explicit form of the improved density-matrix expansion of Gebremariam, Duguet and Bogner [18]. Its Fourier-transform to momentum space provides the adequate technical tool to calculate the nuclear energy density functional in a diagrammatic framework. As a first interesting result we find that for the zero-range Skyrme force the new and the old density-matrix expansion lead to identical results. Differences between the two versions are therefore to be expected for the interaction contributions arising from the long-range $1 \pi$ - and $2 \pi$-exchange between nucleons. In section 3 , we present the analytical results for the densitydependent strength functions $F_{\tau}(\rho), F_{s o}(\rho)$ and $F_{J}(\rho)$ from which the nuclear energy density functional is composed. We restrict ourselves here to the isospin-symmetric case of equal proton and neutron number. These analytical expressions give individually the effects due to $1 \pi$-exchange, iterated $1 \pi$-exchange, and irreducible $2 \pi$-exchange with intermediate $\Delta$-isobar excitations, including Pauli-blocking corrections up to three-loop order. Section 4 is devoted to a discussion of our numerical results and finally section 5 ends with a summary and concluding remarks. In the appendix the three-body spin-orbit coupling strength $F_{s o}(\rho)$ is presented for an alternative description of the $2 \pi$-exchange three-nucleon interaction.

\section{Improved density-matrix expansion and energy den- sity functional}

The starting point for the construction of an explicit nuclear energy density functional is the density-matrix as given by a sum over the occupied energy eigenfunctions $\Psi_{\alpha}$ of the (nonrelativistic) many-fermion system. According to Gebremariam, Duguet and Bogner [18] the bilocal density-matrix can be expanded in relative and center-of-mass coordinates, $\vec{a}$ and $\vec{r}$, as follows: 2

$$
\begin{aligned}
\sum_{\alpha} \Psi_{\alpha}(\vec{r}-\vec{a} / 2) \Psi_{\alpha}^{\dagger}(\vec{r}+\vec{a} / 2)= & \frac{3 \rho}{a k_{f}} j_{1}\left(a k_{f}\right)-\frac{a}{2 k_{f}} j_{1}\left(a k_{f}\right)\left[\tau-\frac{3}{5} \rho k_{f}^{2}-\frac{1}{4} \vec{\nabla}^{2} \rho\right] \\
& +\frac{3 i}{2 a k_{f}} j_{1}\left(a k_{f}\right) \vec{\sigma} \cdot(\vec{a} \times \vec{J})+\ldots,
\end{aligned}
$$

where $j_{1}(x)=(\sin x-x \cos x) / x^{2}$ is the spherical Bessel function of index 1 . The other quantities appearing on the right hand side of eq.(1) are the local nucleon density:

$$
\rho(\vec{r})=\frac{2 k_{f}^{3}(\vec{r})}{3 \pi^{2}}=\sum_{\alpha} \Psi_{\alpha}^{\dagger}(\vec{r}) \Psi_{\alpha}(\vec{r}),
$$

written here in terms of the local Fermi-momentum $k_{f}(\vec{r})$, the local kinetic energy density:

$$
\tau(\vec{r})=\sum_{\alpha} \vec{\nabla} \Psi_{\alpha}^{\dagger}(\vec{r}) \cdot \vec{\nabla} \Psi_{\alpha}(\vec{r})
$$

and the local spin-orbit density:

$$
\vec{J}(\vec{r})=\sum_{\alpha} \Psi_{\alpha}^{\dagger}(\vec{r}) i \vec{\sigma} \times \vec{\nabla} \Psi_{\alpha}(\vec{r}) .
$$

\footnotetext{
${ }^{2}$ We are considering for equal proton and neutron number the spherical phase-space averaged version without quadrupolar deformation of the local Fermi momentum distribution. It brings about already most of the improvements [18.
} 
For notational simplicity we have dropped their argument $\vec{r}$ in eq.(1) and will do so in the following. It is important to note that a pairwise filling of time-reversed orbitals $\alpha$ has been assumed in eq.(1), so that (various possible) time-reversal-odd fields do not come into play [1]. The main difference of this improved density-matrix expansion to the original one of Negele and Vautherin [15] lies in the index of the Bessel function multiplying the kinetic energy and spinorbit densities in eq.(1). The Fourier-transform of the (expanded) density-matrix with respect to both coordinates $\vec{a}$ and $\vec{r}$ defines a "medium insertion" for the inhomogeneous many-nucleon system characterized by the time-reversal-even fields $\rho(\vec{r}), \tau(\vec{r})$ and $\vec{J}(\vec{r})$ :

$$
\begin{aligned}
\Gamma(\vec{p}, \vec{q})= & \int d^{3} r e^{-i \vec{q} \cdot \vec{r}}\left\{\theta\left(k_{f}-|\vec{p}|\right)+\frac{\pi^{2}}{4 k_{f}^{4}}\left[k_{f} \delta^{\prime}\left(k_{f}-|\vec{p}|\right)-2 \delta\left(k_{f}-|\vec{p}|\right)\right]\right. \\
& \left.\times\left(\tau-\frac{3}{5} \rho k_{f}^{2}-\frac{1}{4} \vec{\nabla}^{2} \rho\right)-\frac{3 \pi^{2}}{4 k_{f}^{4}} \delta\left(k_{f}-|\vec{p}|\right) \vec{\sigma} \cdot(\vec{p} \times \vec{J})\right\} .
\end{aligned}
$$

The double line in the left picture of Fig. 1 symbolizes this medium insertion together with the assignment of the out- and in-going nucleon momenta $\vec{p} \pm \vec{q} / 2$. The momentum transfer $\vec{q}$ is provided by the Fourier components of the inhomogeneous (matter) distributions $\rho(\vec{r}), \tau(\vec{r})$ and $\vec{J}(\vec{r})$. As a check one verifies that the Fourier transform $\left(1 / 2 \pi^{3}\right) \int d^{3} p e^{-i \vec{p} \cdot \vec{a}}$ of the expression in the curly brackets in eq.(5) reproduces exactly the right hand side of the (improved) densitymatrix expansion written in eq.(1). In comparison to the version of $\Gamma(\vec{p}, \vec{q})$ which followed from Negele and Vautherin's density-matrix expansion [16] the weight function of the kinetic energy density $\tau(\vec{r})$ has changed from $35\left(5 \vec{p}^{2}-3 k_{f}^{2}\right) \theta\left(k_{f}-|\vec{p}|\right)$ to $2 k_{f}^{3}\left[k_{f} \delta^{\prime}\left(k_{f}-|\vec{p}|\right)-2 \delta\left(k_{f}-|\vec{p}|\right)\right]$ and that of the spin-orbit density $\vec{J}(\vec{r})$ has changed from $\delta\left(k_{f}-|\vec{p}|\right)-k_{f} \delta^{\prime}\left(k_{f}-|\vec{p}|\right)$ to $-3 \delta\left(k_{f}-|\vec{p}|\right)$. For an inhomogeneous many-nucleon system this leads to a different weighting of the momentum dependent nucleon-nucleon interactions in the vicinity of the local Fermi-surface $|\vec{p}|=k_{f}(\vec{r})$, with appropriate consequences for the energy density functional.

Going up to second order in spatial gradients (i.e. deviations from homogeneity) the energy density functional relevant for $N=Z$ even-even nuclei reads:

$$
\begin{aligned}
\mathcal{E}[\rho, \tau, \vec{J}]= & \rho \bar{E}(\rho)+\left[\tau-\frac{3}{5} \rho k_{f}^{2}\right]\left[\frac{1}{2 M}-\frac{k_{f}^{2}}{4 M^{3}}+F_{\tau}(\rho)\right] \\
& +(\vec{\nabla} \rho)^{2} F_{\nabla}(\rho)+\vec{\nabla} \rho \cdot \vec{J} F_{s o}(\rho)+\vec{J}^{2} F_{J}(\rho) .
\end{aligned}
$$

Here, $\bar{E}(\rho)$ is the energy per particle of isospin-symmetric nuclear matter evaluated at the local nucleon density $\rho(\vec{r})$. The (small) correction term $-k_{f}^{2} / 4 M^{3}$ in eq.(6) stems from the relativistically improved kinetic energy and reflects in this way the relativistic increase of mass. The density-dependent functions $F_{\tau}(\rho), F_{\nabla}(\rho), F_{s o}(\rho)$ and $F_{J}(\rho)$ arising from two- and threenucleon interactions encode new dynamical information specific for the inhomogeneous manynucleon system. In particular, $F_{\nabla}(\rho)$ measures the energy associated with density gradients at the nuclear surface and $F_{\nabla}(\rho)$ gives the strength of the spin-orbit coupling.

Returning to eq. (5) one sees that $F_{\tau}(\rho)$ emerges via a perturbation on top of the density of states $\theta\left(k_{f}-|\vec{p}|\right)$. The single-particle potential in nuclear matter can be obtained in the same way by introducing a (three-dimensional) delta-function as the perturbation. Consequently, the strength function $F_{\tau}(\rho)$ can be expressed in terms of the momentum and density-dependent single-particle potential $U\left(p, k_{f}\right)$ as follows:

$$
F_{\tau}(\rho)=\left.\frac{1}{2 k_{f}} \frac{\partial U\left(p, k_{f}\right)}{\partial p}\right|_{p=k_{f}} .
$$



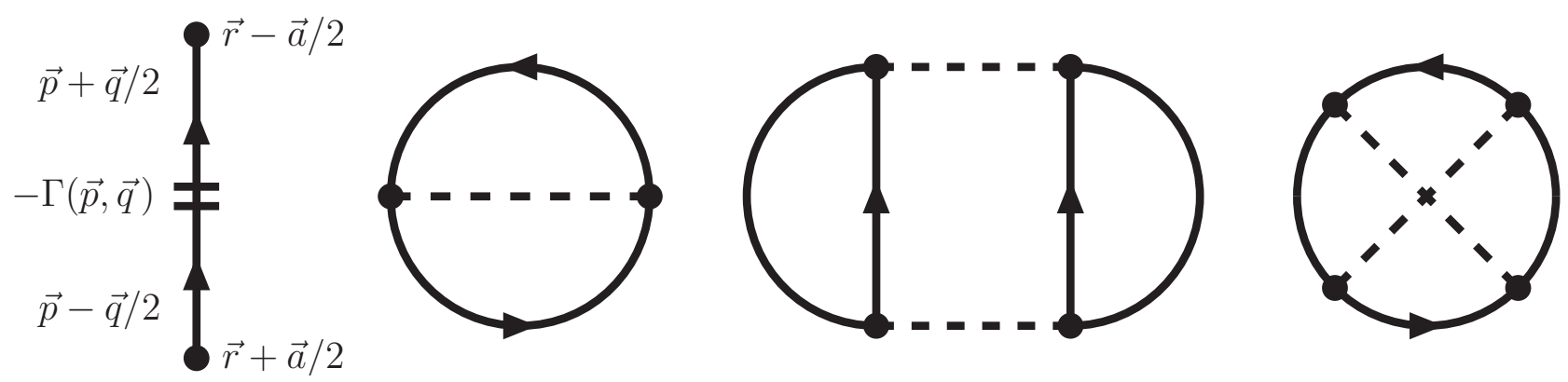

Figure 1: Left: The double line symbolizes the medium insertion defined by eq.(5). Next are shown: The one-pion exchange Fock diagram and the iterated one-pion exchange Hartree and Fock diagrams. Their isospin factors for isospin-symmetric nuclear systems are 6, 12 and -6, respectively.

In eq. (5) the term $\tau-3 \rho k_{f}^{2} / 5$ is accompanied by $-\vec{\nabla}^{2} \rho / 4$. Performing a partial integration of the energy $\int d^{3} r \mathcal{E}[\rho, \tau, \vec{J}]$ one is lead to the decomposition:

$$
F_{\nabla}(\rho)=\frac{1}{4} \frac{\partial F_{\tau}(\rho)}{\partial \rho}+F_{d}(\rho)
$$

where $F_{d}(\rho)$ comprises all those contributions for which the $(\vec{\nabla} \rho)^{2}$-factor originates directly from the momentum dependence of the interactions in an expansion up to order $\vec{q}^{2}$. Since no information about the density-matrix expansion beyond its (fixed) nucleon matter piece $\theta\left(k_{f}-|\vec{p}|\right)$ goes into the derivation of the strength function $F_{d}(\rho)$ the pertinent contributions from the $2 \pi$-exchange dynamics are still given in unchanged form by eqs. $(12,15,19,24,28)$ in ref.[16] and eqs. $(26,30)$ in ref.[17].

As a first test case for the improved density-matrix expansion (summarized in eq.(5)) we have applied it to the (zero-range) Skyrme force [2, 19] and found that it gives identical results:

$$
\begin{aligned}
& F_{\tau}(\rho)^{(\mathrm{Sk})}=\frac{\rho}{16}\left(3 t_{1}+5 t_{2}\right), \quad F_{d}(\rho)^{(\mathrm{Sk})}=\frac{1}{32}\left(3 t_{1}-5 t_{2}\right), \\
& F_{\text {so }}(\rho)^{(\mathrm{Sk})}=\frac{3}{4} W_{0}, \quad F_{J}(\rho)^{(\mathrm{Sk})}=\frac{1}{32}\left(t_{1}-t_{2}\right),
\end{aligned}
$$

for the energy density functional as the original density-matrix expansion of Negele and Vautherin [15]. Obviously, for contact-interactions with their simple quadratic momentum dependence the different weighting of interaction strength in the vicinity of the Fermi-surface has no visible effect. A stronger influence of the actual form of the density-matrix expansion is therefore expected for the contributions arising from the long-range $1 \pi$ - and $2 \pi$-exchange. The pertinent analytical expressions are collected in the next section.

\section{Diagrammatic calculation}

In this section we present analytical formulas for the three density-dependent strength functions $F_{\tau}(\rho), F_{\text {so }}(\rho)$ and $F_{J}(\rho)$ as derived (via the improved density-matrix expansion [18]) from $1 \pi$ exchange, iterated $1 \pi$-exchange, and irreducible $2 \pi$-exchange diagrams with intermediate $\Delta$ isobar excitations, including Pauli-blocking corrections up to three-loop order. We give for each diagram only the final result omitting all technical details related to extensive algebraic manipulations and solving elementary integrals. Further explanations about the organization and performance of our diagrammatic calculation can be found in section 3 of ref. [16]. 


\subsection{One pion exchange Fock diagram with two medium insertions}

The non-vanishing contributions from the $1 \pi$-exchange Fock diagram shown in Fig. 1, including the relativistic $1 / M^{2}$-corrections, read:

$$
\begin{gathered}
F_{\tau}(\rho)=\frac{3 g_{A}^{2} m_{\pi}}{\left(8 \pi f_{\pi}\right)^{2} u^{3}}\left[\left(u^{2}+\frac{1}{2}\right) \ln \left(1+4 u^{2}\right)-2 u^{2}\right] \\
\quad+\frac{3 g_{A}^{2} m_{\pi}^{3}}{\left(8 \pi f_{\pi} M\right)^{2}}\left[4 u-\frac{4 u^{3}}{3}-2 \arctan 2 u-u \ln \left(1+4 u^{2}\right)\right], \\
F_{J}(\rho)=\frac{9 g_{A}^{2}}{\left(32 m_{\pi} f_{\pi}\right)^{2} u^{6}}\left[8 u^{4}-4 u^{2}+\ln \left(1+4 u^{2}\right)\right],
\end{gathered}
$$

where we have introduced the convenient dimensionless variable $u=k_{f} / m_{\pi}$.

\subsection{Iterated one-pion exchange Hartree diagram with two medium insertions}

The two-body contributions from the iterated $1 \pi$-exchange Hartree diagram in Fig. 1 read:

$$
\begin{gathered}
F_{\tau}(\rho)=\frac{g_{A}^{4} M m_{\pi}^{2}}{(8 \pi)^{3} f_{\pi}^{4}}\left\{\frac{7+30 u^{2}}{2 u^{3}} \ln \left(1+4 u^{2}\right)-\frac{14}{u}-16 \arctan 2 u\right\}, \\
F_{s o}(\rho)=\frac{3 g_{A}^{4} M}{\pi m_{\pi}\left(4 f_{\pi} u\right)^{4}}\left\{4 u \arctan 2 u-3 u^{2}-\frac{5}{4} \ln \left(1+4 u^{2}\right)\right\} .
\end{gathered}
$$

The expression for $F_{s o}(\rho)$ in eq.(13) gives (part of) the "wrong-sign" spin-orbit interaction induced by the pion-exchange tensor force in second order. It is completed by the Fock (exchange) contribution and the respective Pauli-blocking corrections (see eqs. $(15,18,22,25)$ ).

\subsection{Iterated one-pion exchange Fock diagram with two medium in- sertions}

We find the following contributions from the right diagram in Fig. 1 with two medium insertions on non-neighboring nucleon propagators:

$$
\begin{aligned}
& F_{\tau}(\rho)= \frac{3 g_{A}^{4} M m_{\pi}^{2}}{\left(4 f_{\pi}\right)^{4}(\pi u)^{3}} \int_{0}^{u} d x \frac{2 x^{2}-u^{2}}{1+2 x^{2}} \\
& \times\left[\left(1+8 x^{2}+8 x^{4}\right) \arctan x-\left(1+4 x^{2}\right) \arctan 2 x\right], \\
& F_{\text {so }}(\rho)= \frac{3 g_{A}^{4} M}{2 \pi m_{\pi}\left(4 f_{\pi} u\right)^{4}}\left\{u^{2}+\int_{0}^{u} d x \frac{1}{1+2 x^{2}}\right. \\
&\left.\times\left[4 x^{2}\left(1+x^{2}\right) \arctan x-\left(1+4 x^{2}\right) \arctan 2 x\right]\right\}, \\
& F_{J}(\rho)=\frac{9 g_{A}^{4} M}{\pi m_{\pi}\left(8 f_{\pi} u\right)^{4}}\left\{\frac { 2 } { u ^ { 2 } } \int _ { 0 } ^ { u } d x \frac { 1 } { 1 + 2 x ^ { 2 } } \left[\left(u^{2}-x^{2}\right)\left(1+4 x^{2}\right) \arctan 2 x\right.\right. \\
&+\left.\left.2\left(u^{2}-2 x^{2}+2 u^{2} x^{2}-6 x^{4}+2 u^{2} x^{4}-6 x^{6}\right) \arctan x\right]-u^{2}\right\} .
\end{aligned}
$$




\subsection{Iterated one-pion exchange Hartree diagram with three medium insertions}

In our way of organizing the many-body calculation, the Pauli-blocking corrections are represented by diagrams with three medium insertions. The corresponding contributions from the iterated $1 \pi$-exchange Hartree diagram read:

$$
\begin{aligned}
F_{\tau}(\rho)= & \frac{3 g_{A}^{4} M m_{\pi}^{2}}{\left(4 \pi f_{\pi}\right)^{4}}\left\{2 u^{2}-\ln \left(1+4 u^{2}\right)+\frac{2 u^{2}}{1+4 u^{2}}+2 \int_{0}^{1} d y y^{2} \ln \frac{1+y}{1-y}\right. \\
& \times\left[\frac{8 u^{4} y^{2}}{\left(1+4 u^{2} y^{2}\right)^{2}}\left(6 u^{2} y^{2}+y^{2}-2 u^{2}\right)-4 u^{2} y^{2}+\ln \left(1+4 u^{2} y^{2}\right)\right] \\
& +\int_{0}^{u} d x \frac{x^{2}}{u^{3}} \int_{-1}^{1} d y\left[\frac{2 u x y}{u^{2}-x^{2} y^{2}}+\ln \frac{u+x y}{u-x y}\right]\left[2 \ln \left(1+s^{2}\right)\right. \\
& \left.\left.-\frac{2 s^{2}+s^{4}}{1+s^{2}}+\frac{\left(u^{2}-x^{2} y^{2}\right) s^{4}}{u^{2}\left(1+s^{2}\right)^{3}}\left(\left(5+s^{2}\right) s^{2}+\left(s+s^{3}\right)\left(s^{\prime \prime}-2 s^{\prime}\right)\right)\right]\right\},
\end{aligned}
$$

with the auxiliary function $s=x y+\sqrt{u^{2}-x^{2}+x^{2} y^{2}}$, and its partial derivatives $s^{\prime}=u \partial s / \partial u$ and $s^{\prime \prime}=u^{2} \partial^{2} s / \partial u^{2}$.

$$
\begin{aligned}
F_{\text {so }}(\rho)= & \frac{3 g_{A}^{4} M}{\pi^{2} m_{\pi}\left(4 f_{\pi}\right)^{4}} \int_{0}^{u} d x \frac{x^{2}}{u^{6}} \int_{-1}^{1} d y\left\{\left[4 x y \ln \frac{u+x y}{u-x y}+\frac{u\left(5 x^{2} y^{2}-3 u^{2}\right)}{u^{2}-x^{2} y^{2}}\right]\right. \\
& \times\left[5 s+\frac{s}{\left(1+s^{2}\right)^{2}}-6 \arctan s\right]-\frac{u s^{5}\left(u^{2}+x^{2} y^{2}\right)}{\left(1+s^{2}\right)^{2}\left(u^{2}-x^{2} y^{2}\right)} \\
& \left.+\frac{2 s^{4} s^{\prime}(s-2 x y)}{\left(1+s^{2}\right)^{2}} \ln \frac{u+x y}{u-x y}\right\}, \\
F_{J}(\rho) & =\frac{9 g_{A}^{4} M u^{3}}{16 \pi^{2} m_{\pi} f_{\pi}^{4}} \int_{0}^{1} d y \frac{y^{6}}{\left(1+4 u^{2} y^{2}\right)^{2}}\left[2 y+\left(1-y^{2}\right) \ln \frac{1+y}{1-y}\right] .
\end{aligned}
$$

\subsection{Iterated one-pion exchange Fock diagram with three medium insertions}

The evaluation of this diagram is most tedious. It is advisable to split the contributions to the strength functions $F_{\tau}(\rho), F_{s o}(\rho)$ and $F_{J}(\rho)$ into "factorizable" and "non-factorizable" parts. These two pieces are distinguished by the feature of whether the nucleon propagator in the denominator can be canceled or not by terms from the product of $\pi N$-interaction vertices in the numerator. We find the following "factorizable" contributions:

$$
\begin{aligned}
F_{\tau}(\rho)= & \frac{3 g_{A}^{4} M m_{\pi}^{2}}{\left(4 \pi f_{\pi} u\right)^{4}}\left\{\frac{1}{8}\left(1+4 u^{2}+2 u^{4}\right) \ln \left(1+4 u^{2}\right)-\frac{1+6 u^{2}+8 u^{4}}{64 u^{2}} \ln ^{2}\left(1+4 u^{2}\right)\right. \\
& -\frac{u^{4}}{2}-\frac{u^{2}}{4}+\frac{u}{2} \int_{0}^{u} d x\left[u\left(1+u^{2}+x^{2}\right)-\left(1+(u+x)^{2}\right)\left(1+(u-x)^{2}\right) L\right] \\
& \left.\times\left[\left(1-u^{2}-x^{2}\right) L+u-\frac{u}{1+(u+x)^{2}}-\frac{u}{1+(u-x)^{2}}\right]\right\},
\end{aligned}
$$

with the auxiliary function:

$$
L(x, u)=\frac{1}{4 x} \ln \frac{1+(u+x)^{2}}{1+(u-x)^{2}}
$$




$$
\begin{aligned}
F_{\text {so }}(\rho)= & \frac{3 g_{A}^{4} M}{\pi^{2} m_{\pi}\left(8 f_{\pi} u\right)^{4}}\left\{4\left[\ln \left(1+4 u^{2}\right)-7 u^{2}\right] \arctan 2 u+28 u^{3}+8 u+\frac{3}{u}\right. \\
& -\frac{3+14 u^{2}+10 u^{4}}{2 u^{3}} \ln \left(1+4 u^{2}\right)+\frac{3+20 u^{2}+16 u^{4}}{16 u^{5}} \ln ^{2}\left(1+4 u^{2}\right) \\
& +4 \int_{0}^{u} d x\left\{L^{2}\left[3 x^{-2}\left(1+u^{2}\right)^{3}+3+2 u^{2}-u^{4}-\left(3+7 u^{2}\right) x^{2}+5 x^{4}\right]\right. \\
& \left.\left.-6 u x^{-2}\left(1+u^{2}\right)^{2} L+3 u^{2} x^{-2}\left(1+u^{2}\right)\right\}\right\}, \\
F_{J}(\rho)= & \frac{9 g_{A}^{4} M}{\pi^{2} m_{\pi}\left(8 f_{\pi} u\right)^{4}}\left\{7 \arctan 2 u+4 u^{3}-\frac{1}{u}-\frac{1+4 u^{2}}{16 u^{5}} \ln ^{2}\left(1+4 u^{2}\right)\right. \\
& -\frac{37 u}{4}+\frac{8-7 u^{2}-12 u^{4}}{16 u^{3}} \ln \left(1+4 u^{2}\right)+\int_{0}^{u} d x\left\{\frac { L ^ { 2 } } { u ^ { 2 } } \left[\frac{3}{2 x^{2}}\left(1+u^{2}\right)^{4}\right.\right. \\
& \left.+2\left(1-u^{4}\right)\left(1+u^{2}\right)+\left(5+2 u^{2}+5 u^{4}\right) x^{2}-\left(6+10 u^{2}\right) x^{4}+\frac{11 x^{6}}{2}\right] \\
& \left.\left.+\frac{L}{u}\left[3 u^{4}+2 u^{2}-1-\frac{3}{x^{2}}\left(1+u^{2}\right)^{3}\right]+\frac{3}{2 x^{2}}\left(1+u^{2}\right)^{2}\right\}\right\} .
\end{aligned}
$$

The "non-factorizable" contributions (stemming from nine-dimensional principal value integrals over the product of three Fermi-spheres of radius $k_{f}$ ) read on the other hand:

$$
\begin{gathered}
F_{\tau}(\rho)=\frac{3 g_{A}^{4} M m_{\pi}^{2}}{\left(4 \pi f_{\pi}\right)^{4}} \int_{-1}^{1} d y \int_{-1}^{1} d z \frac{y z \theta\left(y^{2}+z^{2}-1\right)}{|y z| \sqrt{y^{2}+z^{2}-1}}\left\{\frac{4 u^{2} z^{2}\left(2 z^{2}-1\right)}{1+4 u^{2} z^{2}}\right. \\
\quad \times\left[\ln \left(1+4 u^{2} y^{2}\right)-4 u^{2} y^{2}\right] \theta(y) \theta(z)+\int_{0}^{u} d x \frac{x^{2} s^{2}}{2 u^{5}\left(1+s^{2}\right)^{2}} \\
\left.\times\left[t^{2}-\ln \left(1+t^{2}\right)\right]\left[\left(s+s^{3}\right)\left(2 s^{\prime}-s^{\prime \prime}\right)-\left(3+s^{2}\right) s^{\prime 2}\right]\right\}, \\
F_{s o}(\rho)=\frac{3 g_{A}^{4} M}{\pi^{2} m_{\pi}\left(4 f_{\pi}\right)^{4}} \int_{-1}^{1} d y \int_{-1}^{1} d z \frac{y z \theta\left(y^{2}+z^{2}-1\right)}{|y z| \sqrt{y^{2}+z^{2}-1}\left\{\frac{8 y^{2} z \theta(y) \theta(z)}{1+4 u^{2} y^{2}}\right.} \\
\left.\times[\arctan (2 u z)-2 u z]+\int_{0}^{u} d x \frac{x^{2} s^{2} s^{\prime} t^{2} t^{\prime}}{2 u^{8}\left(1+s^{2}\right)\left(1+t^{2}\right)}(t x y-s x z-s t)\right\}, \\
F_{J}(\rho)=\frac{9 g_{A}^{4} M}{\pi^{2} m_{\pi}\left(4 f_{\pi}\right)^{4}} \int_{-1}^{1} d y \int_{-1}^{1} d z \frac{y z \theta\left(y^{2}+z^{2}-1\right)}{|y z| \sqrt{y^{2}+z^{2}-1}}\left\{\frac{2 y^{4} \theta(y) \theta(z)}{u\left(1+4 u^{2} y^{2}\right)}\right. \\
\left.\quad \times\left[\ln \left(1+4 u^{2} z^{2}\right)-4 u^{2} z^{2}\right]+\int_{0}^{u} d x \frac{x^{4} s^{3} s^{\prime} t^{3} t^{\prime}\left(1-y^{2}-z^{2}\right)}{4 u^{10}\left(1+s^{2}\right)\left(1+t^{2}\right)}\right\},
\end{gathered}
$$

with the auxiliary function $t=x z+\sqrt{u^{2}-x^{2}+x^{2} z^{2}}$ and its partial derivative $t^{\prime}=u \partial t / \partial u$. For the numerical evaluation of the $d y d z$-double integrals in eqs. $(24,25,26)$ it is advantageous to first antisymmetrize the integrands in $y$ and $z$ and then to substitute $z=\sqrt{y^{2} \zeta^{2}+1-y^{2}}$. This way the integration region becomes equal to the unit-square $0<y, \zeta<1$.

\subsection{Irreducible two-pion exchange}

At next order in the small momentum expansion comes the irreducible $2 \pi$-exchange including (also) intermediate $\Delta$-isobar excitations. We employ a (subtracted) spectral-function represen- 
tation of the $\pi N \Delta$-loops and find the following (two-body) contributions:

$$
\begin{gathered}
F_{\tau}(\rho)=\frac{1}{8 \pi^{3}} \int_{2 m_{\pi}}^{\infty} d \mu \operatorname{Im}\left(V_{C}+3 W_{C}+2 \mu^{2} V_{T}+6 \mu^{2} W_{T}\right) \\
\times\left[\frac{2 \mu}{k_{f}}+\frac{8 k_{f}^{3}}{3 \mu^{3}}-\frac{\mu}{2 k_{f}^{3}}\left(\mu^{2}+2 k_{f}^{2}\right) \ln \left(1+\frac{4 k_{f}^{2}}{\mu^{2}}\right)\right], \\
F_{J}(\rho)=\frac{3}{16 \pi} \int_{2 m_{\pi}}^{\infty} d \mu\left\{\operatorname{Im}\left(V_{C}+3 W_{C}\right)\left[\frac{\mu}{4 k_{f}^{6}}\left(\mu^{2}+2 k_{f}^{2}\right) \ln \left(1+\frac{4 k_{f}^{2}}{\mu^{2}}\right)-\frac{\mu}{k_{f}^{4}}-\frac{4}{3 \mu^{3}}\right]\right. \\
\left.+\operatorname{Im}\left(V_{T}+3 W_{T}\right)\left[\frac{\mu}{k_{f}^{2}}-\frac{4}{3 \mu}+\frac{\mu^{3}}{2 k_{f}^{4}}-\frac{\mu^{3}}{8 k_{f}^{6}}\left(\mu^{2}+4 k_{f}^{2}\right) \ln \left(1+\frac{4 k_{f}^{2}}{\mu^{2}}\right)\right]\right\} .
\end{gathered}
$$

The imaginary parts $\operatorname{Im} V_{C}, \operatorname{Im} W_{C}, \operatorname{Im} V_{T}$ and $\operatorname{Im} W_{T}$ of the isoscalar and isovector central and tensor $\mathrm{NN}$-amplitudes due to $2 \pi$-exchange with single and double $\Delta$-excitation can be found in section 3 of ref.[20]. The additional contributions from the irreducible $2 \pi$-exchange with only nucleon intermediate states are accounted for by inserting into eqs. $(27,28)$ the imaginary parts:

$$
\begin{gathered}
\operatorname{Im} W_{C}=\frac{\sqrt{\mu^{2}-4 m_{\pi}^{2}}}{3 \pi \mu\left(4 f_{\pi}\right)^{4}}\left[4 m_{\pi}^{2}\left(1+4 g_{A}^{2}-5 g_{A}^{4}\right)+\mu^{2}\left(23 g_{A}^{4}-10 g_{A}^{2}-1\right)+\frac{48 g_{A}^{4} m_{\pi}^{4}}{\mu^{2}-4 m_{\pi}^{2}}\right], \\
\operatorname{Im} V_{T}=-\frac{6 g_{A}^{4} \sqrt{\mu^{2}-4 m_{\pi}^{2}}}{\pi \mu\left(4 f_{\pi}\right)^{4}} .
\end{gathered}
$$

At leading order the irreducible $2 \pi$-exchange generates no spin-orbit NN-interaction. It emerges first as a relativistic $1 / M$-correction. In order to see the size of such relativistic effects we have evaluated the energy density functional with a two-body interaction composed of the (isoscalar and isovector) spin-orbit NN-amplitudes $V_{\mathrm{SO}}$ and $W_{\mathrm{SO}}$ written in eqs.(22,23) of ref.[21]. We find with it the following contribution to the spin-orbit coupling strength:

$$
\begin{aligned}
F_{\text {so }}(\rho)= & \frac{g_{A}^{2} m_{\pi}}{\pi M\left(4 f_{\pi}\right)^{4}}\left\{\left(g_{A}^{2}-\frac{4}{5}\right)\left[\frac{1}{u^{4}} \ln \left(1+u^{2}\right)-\frac{1}{u^{2}}\right]\right. \\
& \left.+\frac{18}{5}-\frac{3 g_{A}^{2}}{2}+\left(\frac{2 g_{A}^{2}-4}{u}-\frac{12 u}{5}\right) \arctan u\right\},
\end{aligned}
$$

which has been subtracted at $\rho=0$ in order to eliminate (regularization dependent) shortdistance components. As a consequence of that subtraction only the Fock terms are included in the expressions in eqs. $(27,28,31)$.

\subsection{Three-body diagrams with $\Delta$-excitation}

The Pauli-blocking correction to the $2 \pi$-exchange with single $\Delta$-excitation is equivalent to the contribution of a (genuine) three-nucleon force. In fact, one is dealing here with the same threenucleon interaction as originally introduced by Fujita and Miyazawa [22]. Moreover, it has been shown in ref. 13] that the inclusion of this long-range $2 \pi$-exchange three-nucleon interaction is essential in order to reproduce the empirical saturation point of nuclear matter when using the low-momentum NN-potential $V_{\text {low-k }}$ in Hartree-Fock calculations. It is therefore equally interesting to see its effects on the nuclear energy functional $\mathcal{E}[\rho, \tau, \vec{J}]$.

The pertinent Hartree and Fock three-body diagrams related to $2 \pi$-exchange with virtual $\Delta$-excitation are shown in Fig. 2. The central diagram with parallel pion-lines vanishes for 

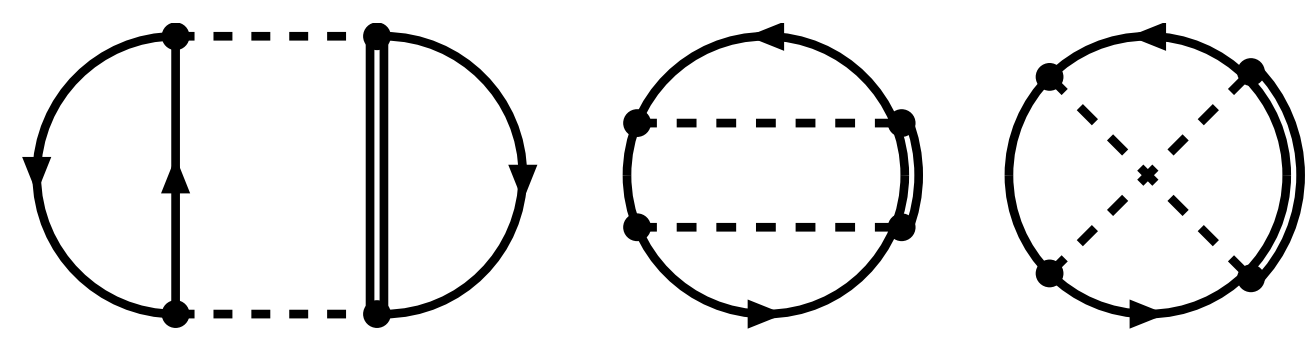

Figure 2: Hartree and Fock three-body diagrams related to $2 \pi$-exchange with virtual $\Delta$-isobar excitation. Their isospin factors for isospin-symmetric nuclear systems are 8,0 , and 8 , respectively.

isospin-symmetric nuclear systems. Returning to the medium insertion written in eq.(5) we find from the left three-body Hartree diagram in Fig. 2 the following contributions:

$$
\begin{gathered}
F_{\tau}(\rho)=\frac{g_{A}^{4} m_{\pi}^{4}}{\Delta\left(2 \pi f_{\pi}\right)^{4}}\left\{\left(u^{2}+\frac{3}{4}\right) \ln \left(1+4 u^{2}\right)-\frac{u^{2}\left(3+10 u^{2}\right)}{1+4 u^{2}}\right\}, \\
F_{s o}(\rho)=\frac{3 g_{A}^{4} m_{\pi}}{\pi^{2} \Delta\left(4 f_{\pi}\right)^{4}}\left\{\frac{12}{u}+8 u-\frac{3+8 u^{2}}{u^{3}} \ln \left(1+4 u^{2}\right)\right\}, \\
F_{J}(\rho)=\frac{3 g_{A}^{4} m_{\pi}}{\pi^{2} \Delta\left(4 f_{\pi}\right)^{4}}\left\{\frac{3}{2 u^{3}} \ln \left(1+4 u^{2}\right)+4 u-\frac{6}{u}+\frac{8 u}{1+4 u^{2}}\right\},
\end{gathered}
$$

with $\Delta=293 \mathrm{MeV}$ the delta-nucleon mass splitting. We have used the value $3 / \sqrt{2}$ for the ratio between the $\pi N \Delta$ - and $\pi N N$-coupling constants. Note that the expression for $F_{\text {so }}(\rho)$ in eq.(33) gives the (dominant part of the) three-body spin-orbit coupling strength suggested originally by Fujita and Miyazawa 22. The three-body effects on the energy density functional are completed by the contributions from the right Fock diagram in Fig. 2 which read:

$$
\begin{aligned}
F_{\tau}(\rho)= & \frac{g_{A}^{4} m_{\pi}^{4}}{\Delta\left(4 \pi f_{\pi}\right)^{4}}\left\{\frac{2}{u^{3}}\left[4 u^{2}-\left(1+2 u^{2}\right) \ln \left(1+4 u^{2}\right)\right] \arctan 2 u-\frac{2 u^{4}}{3}\right. \\
& +\frac{31 u^{2}}{6}-\frac{31}{4}+\frac{5}{8 u^{2}}+\frac{3+22 u^{2}+176 u^{4}+288 u^{6}}{256 u^{8}} \ln ^{2}\left(1+4 u^{2}\right) \\
& +\frac{3}{16 u^{4}}+\frac{1}{96 u^{6}}\left(248 u^{6}-224 u^{8}-60 u^{4}-48 u^{2}-9\right) \ln \left(1+4 u^{2}\right) \\
& +\frac{1}{u^{3}} \int_{0}^{u} d x\left\{G_{S}\left[\frac{2 u(u+x)}{1+(u+x)^{2}}+\frac{2 u(x-u)}{1+(u-x)^{2}}-4 x L\right]\right. \\
& +G_{T}\left[\frac{3 u}{4 x}\left(3 u^{2}-1\right)-\frac{3 u x}{4}+\frac{u(u+x)}{1+(u+x)^{2}}+\frac{u(x-u)}{1+(u-x)^{2}}\right. \\
& \left.\left.\left.+\frac{L}{4 x}\left(3 x^{4}+6 u^{2} x^{2}-2 x^{2}-9 u^{4}-6 u^{2}+3\right)\right]\right\}\right\}
\end{aligned}
$$

with the auxiliary functions:

$$
\begin{aligned}
G_{S}(x, u)= & \frac{4 u x}{3}\left(2 u^{2}-3\right)+4 x[\arctan (u+x)+\arctan (u-x)] \\
& +\left(x^{2}-u^{2}-1\right) \ln \frac{1+(u+x)^{2}}{1+(u-x)^{2}}
\end{aligned}
$$




$$
\begin{aligned}
& G_{T}(x, u)=\frac{u x}{6}\left(8 u^{2}+3 x^{2}\right)-\frac{u}{2 x}\left(1+u^{2}\right)^{2} \\
& +\frac{1}{8}\left[\frac{\left(1+u^{2}\right)^{3}}{x^{2}}-x^{4}+\left(1-3 u^{2}\right)\left(1+u^{2}-x^{2}\right)\right] \ln \frac{1+(u+x)^{2}}{1+(u-x)^{2}} \text {. } \\
& F_{\text {so }}(\rho)=\frac{g_{A}^{4} m_{\pi}}{\pi^{2} \Delta\left(8 f_{\pi}\right)^{4}}\left\{\frac{64 u}{3}-\frac{4}{u}-\frac{7}{u^{3}}-\frac{12}{u^{5}}-\frac{15}{4 u^{7}}\right. \\
& +\left(\frac{15}{8 u^{9}}+\frac{39}{4 u^{7}}+\frac{13}{u^{5}}+\frac{6}{u^{3}}-\frac{8}{u}\right) \ln \left(1+4 u^{2}\right) \\
& \left.-\frac{3}{64 u^{11}}\left(64 u^{6}+80 u^{4}+36 u^{2}+5\right) \ln ^{2}\left(1+4 u^{2}\right)\right\} \text {, } \\
& F_{J}(\rho)=\frac{g_{A}^{4} m_{\pi}}{\pi^{2} \Delta\left(8 f_{\pi} u\right)^{4}}\left\{24\left[4-8 u^{2}-\frac{1}{u^{2}} \ln \left(1+4 u^{2}\right)\right] \arctan 2 u-144 u^{5}+\frac{3}{u}\right. \\
& +272 u^{3}-99 u+\frac{9}{4 u^{3}}+\left(28 u-\frac{15}{4 u^{3}}-\frac{9}{8 u^{5}}\right) \ln \left(1+4 u^{2}\right)+\frac{3}{64 u^{7}} \\
& \times\left(3+16 u^{2}+144 u^{4}\right) \ln ^{2}\left(1+4 u^{2}\right)+\frac{3}{4}\left(58 u^{4}+31 u^{2}-63\right) \arctan 2 u \\
& +\frac{663 u^{3}}{16}+\frac{495 u}{16}-\frac{656 u^{5}}{5}+\frac{9}{64 u}\left(29-229 u^{2}+52 u^{4}\right) \ln \left(1+4 u^{2}\right) \\
& +\int_{0}^{u} d x\left\{\frac { 9 L ^ { 2 } } { 8 u ^ { 2 } } \left[\frac{6}{x^{2}}\left(1+u^{2}\right)^{4}\left(3 u^{2}-1\right)-\frac{5}{x^{4}}\left(1+u^{2}\right)^{6}+\left(1+u^{2}\right)^{2}\right.\right. \\
& \times\left(50 u^{2}-39-55 u^{4}\right)+4 x^{2}\left(35 u^{6}+5 u^{4}-39 u^{2}-9\right)-33 x^{8} \\
& \left.+13 x^{4}\left(1+2 u^{2}-15 u^{4}\right)+2 x^{6}\left(65 u^{2}-11\right)\right]+\frac{3 L}{4 u}\left[\frac{15}{x^{4}}\left(1+u^{2}\right)^{5}\right. \\
& \left.+\frac{1}{x^{2}}\left(1+u^{2}\right)^{3}\left(3-49 u^{2}\right)+6\left(25 u^{6}+5 u^{4}-u^{2}+19\right)\right] \\
& \left.\left.-\frac{45}{8 x^{4}}\left(1+u^{2}\right)^{4}+\frac{3}{2 x^{2}}\left(1+u^{2}\right)^{2}\left(3+11 u^{2}\right)\right\}\right\} \text {. }
\end{aligned}
$$

In the appendix we present the three-body spin-orbit coupling strength $F_{\text {so }}(\rho)$ for an alternative description of the $2 \pi$-exchange three-nucleon interaction (using $\pi \pi N N$-contact vertices instead of propagating $\Delta$-isobars). A good check of all formulas collected in this section is provided by their Taylor series expansion in $k_{f}$. Despite the superficial opposite appearance the leading term in the $k_{f}$-expansion is always a non-negative power of $k_{f}$ (which is higher for three-body contributions than for two-body contributions).

\section{Results and discussion}

In this section we present and discuss our numerical results obtained by summing the series of contributions given in section 3 . The physical input parameters are: $g_{A}=1.3$ (nucleon axial vector coupling constant), $f_{\pi}=92.4 \mathrm{MeV}$ (pion decay constant), $m_{\pi}=135 \mathrm{MeV}$ (neutral pion mass) and $M=939 \mathrm{MeV}$ (nucleon mass). We recall that with these physical parameters and a few adjustable short-distance couplings the nuclear matter equation of state $\bar{E}(\rho)$ and many other nuclear matter properties [17] can be well described by the chiral pion-nucleon dynamics treated to three-loop order. 


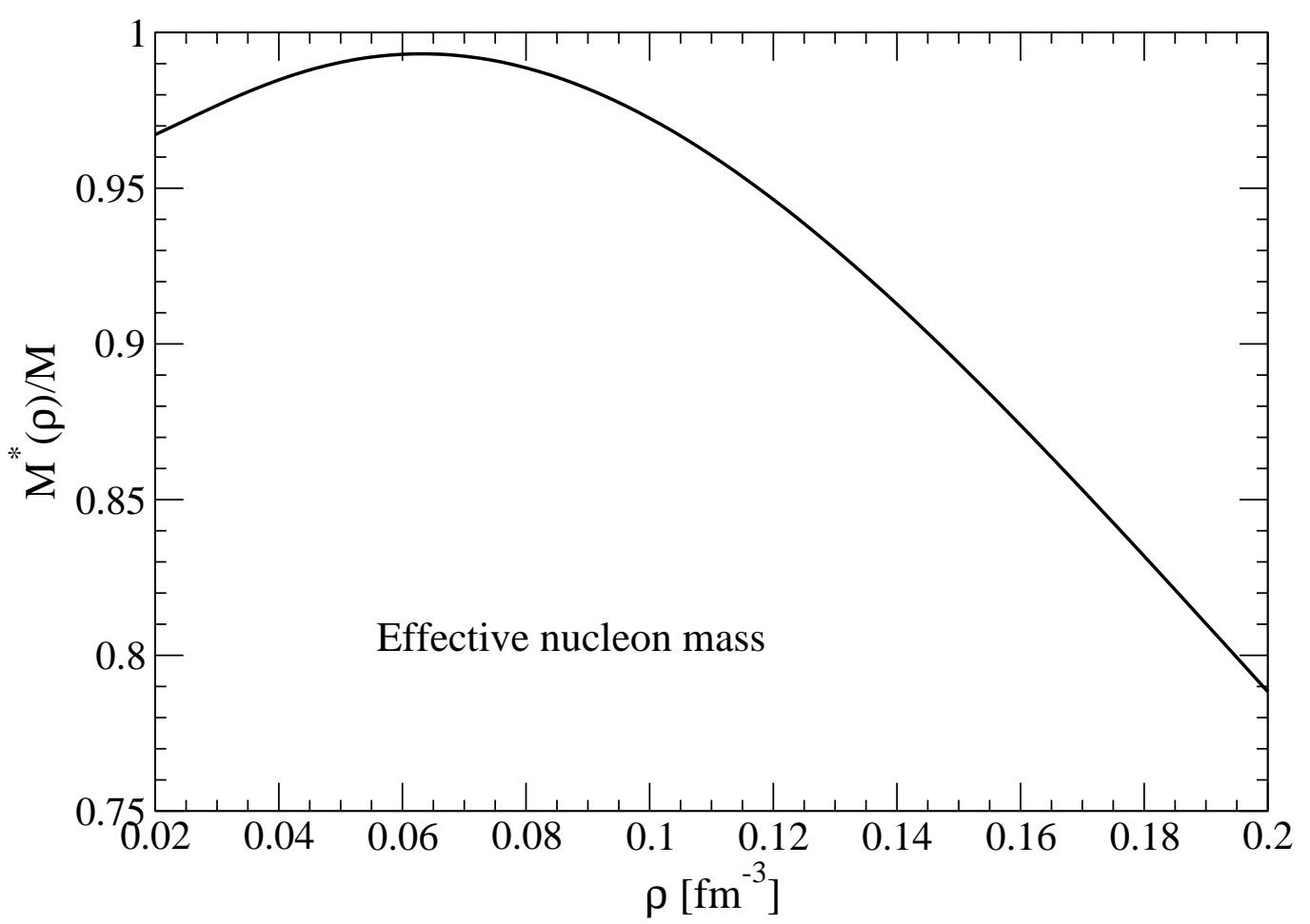

Figure 3: The effective nucleon mass $M^{*}(\rho)$ divided by the free nucleon mass $M$ as a function of the nuclear density $\rho$.

Returning to the energy density functional $\mathcal{E}[\rho, \tau, \vec{J}]$ in eq.(6) one observes that the expression multiplying the kinetic energy density $\tau(\vec{r})$ has the meaning of a reciprocal densitydependent effective nucleon mass:

$$
M^{*}(\rho)=M\left[1-\frac{k_{f}^{2}}{2 M^{2}}+2 M F_{\tau}(\rho)\right]^{-1} .
$$

It is identical to the so-called "Landau" mass introduced in Fermi-liquid theory, since it derives in the same way from the slope of the single-particle potential $U\left(p, k_{f}\right)$ at the Fermi-surface $p=k_{f}$. This consistency of effective nucleon masses follows from the improved density-matrix expansion of Gebremariam, Duguet and Bogner [18], but it did not hold for the original densitymatrix expansion of Negele and Vautherin [15, 16].

Fig. 3 shows the ratio of effective to free nucleon mass $M^{*}(\rho) / M$ as a function of the nuclear density $\rho=2 k_{f}^{3} / 3 \pi^{2}$. One observes a reduced effective nucleon mass which reaches the value $M^{*}\left(\rho_{0}\right)=0.874 M$ at nuclear matter saturation density $\rho_{0}=0.16 \mathrm{fm}^{-3}$. This is compatible with the range $0.7<M^{*}\left(\rho_{0}\right) / M<1$ spanned by phenomenological Skyrme forces [3, 4, 5, 6]. Somewhat unusual is the non-monotonic progression of the curve in Fig. 3. It reveals that a sufficiently high density $\left(0.4 \rho_{0}\right)$ has to be reached until the subleading $\pi N \Delta$-dynamics can revert the tendency of the iterated $1 \pi$-exchange to increase the effective nucleon mass. The same feature has also been observed for the $p$-wave Landau parameter $f_{1}\left(k_{f}\right)$ in ref.[23] (see Fig. 3 therein), a quantity which is intimately related to the effective nucleon mass $M^{*}(\rho)$.

Next, we show in Fig. 4 the strength function $F_{\nabla}(\rho)$ belonging to the $(\vec{\nabla} \rho)^{2}$ surface-term. The dashed line corresponds to the truncation to $1 \pi$ - and iterated $1 \pi$-exchange, whereas the full line includes in addition the $2 \pi$-exchange and the associated three-body contributions. Taking the band spanned by phenomenological Skyrme forces [3, 4, 5, 6] as a benchmark one may conclude that the subleading $2 \pi$-exchange dynamics leads to some improvement. The 


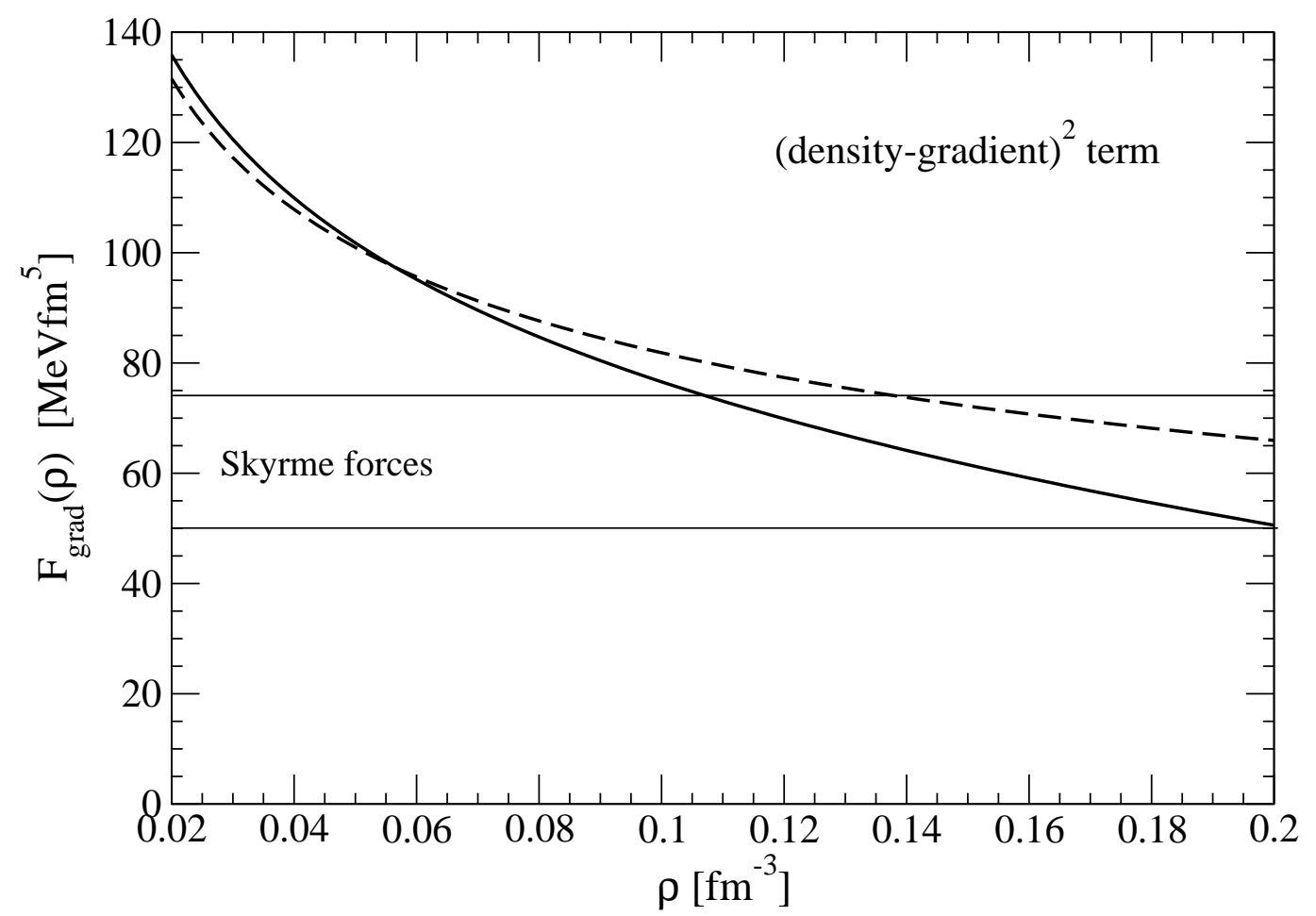

Figure 4: The strength function $F_{\nabla}(\rho)$ of the surface-term $(\vec{\nabla} \rho)^{2}$ in the nuclear energy density functional versus the nuclear density $\rho$. The dashed line corresponds to the truncation to $1 \pi-$ and iterated $1 \pi$-exchange. The full line includes also $2 \pi$-exchange and associated three-body contributions.

improved density-matrix expansion [18] has furthermore flattened and shifted downward the curve for $F_{\nabla}(\rho)$ in comparison to our previous calculation (see Fig. 8 in ref.[17]) based on the Negele-Vautherin density-matrix expansion. We also note that in the relevant density region $\rho_{0} / 2<\rho<\rho_{0}$ the main contribution to the strength function $F_{\nabla}(\rho)$ comes from the component $F_{d}(\rho)$ (see eq. $(8)$ ) which is insensitive to the density-matrix expansion beyond its fixed nuclear matter part $\theta\left(k_{f}-|\vec{p}|\right)$.

Of particular interest is the strength $F_{\text {so }}(\rho)$ of the spin-orbit coupling provided by the explicit pion-exchange dynamics. The dashed curve in Fig. 5 shows the "wrong-sign" spin-orbit coupling strength arising from iterated $1 \pi$-exchange (i.e. the pion-exchange tensor force in second order). Its value at half nuclear matter density $\rho_{0} / 2=0.08 \mathrm{fm}^{-3}$ decomposes as $((-124.4+47.7)+$ $(76.5-35.0)) \mathrm{MeVfm}^{5}=-35.1 \mathrm{MeVfm}^{5}$ into Hartree and Fock pieces supplemented by the respective Pauli-blocking corrections. This net negative result amounts to about $-40 \%$ of the empirical spin-orbit coupling strength $F_{s o}^{(\mathrm{emp})} \simeq 90 \mathrm{MeVfm}^{5}$. In comparison to our previous calculation [16] based on the Negele-Vautherin density-matrix expansion which gave at $\rho_{0} / 2$ the value $-58.1 \mathrm{MeVfm}^{5}$ the "wrong-sign" spin-orbit coupling strength has substantially decreased in magnitude. The full line in Fig. 5 shows the spin-orbit coupling strength after including the subleading $2 \pi$-exchange, in particular the three-body contributions eqs.(33,38). One finds now a pronounced cancellation in the density region around $\rho_{0} / 2=0.08 \mathrm{fm}^{-3}$, where the spin-orbit interaction in nuclei gains actually most of its weight. Such an almost complete cancellation leaves then all room for the short-distance NN-dynamics (not treated explicitly in this work) to account for the strong spin-orbit coupling in nuclei. In fact, it has been shown in ref. [24] that the empirical value $F_{\text {so }}^{(\mathrm{emp})} \simeq 90 \mathrm{MeVfm}^{5}$ of the spin-orbit coupling strength in nuclei is in perfect agreement with the one extracted from realistic nucleon-nucleon potentials. The 


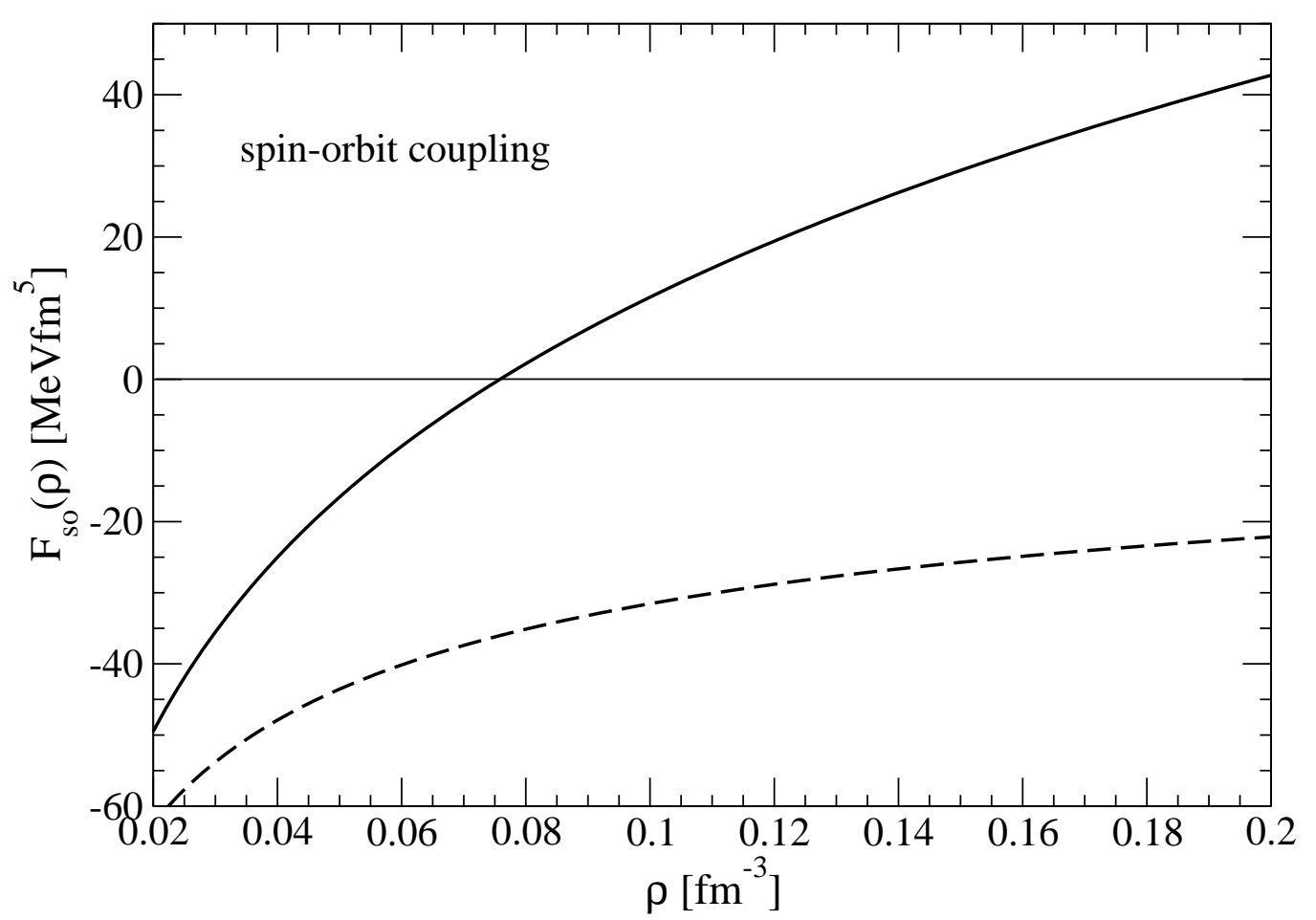

Figure 5: The strength function $F_{\text {so }}(\rho)$ of the spin-orbit coupling term $\vec{\nabla} \rho \cdot \vec{J}$ in the nuclear energy density functional versus the nuclear density $\rho$. Dashed line: $1 \pi$ - and iterated $1 \pi$ exchange only. Full line: $2 \pi$-exchange and three-body contributions added.

intimate connection between the strong Lorentz scalar and vector mean-fields and the (shortrange) spin-orbit part of the NN-potential has been elucidated in ref. 25] via (relativistic) DiracBrueckner calculations of the in-medium nucleon self-energy.

Moreover, we note that the spin-orbit coupling strength generated by the irreducible $2 \pi$ exchange as a relativistic $1 / M$-correction (see eq.(31) in section 3.6) contributes little to the cancellation between "wrong-sign" and "correct-sign" parts shown in Fig. 5 . At $\rho_{0} / 2=0.08 \mathrm{fm}^{-3}$ this piece amounts to just about $-4.0 \mathrm{MeVfm}^{5}$. Furthermore, we have convinced ourselves that the spin-orbit NN-amplitudes from $2 \pi$-exchange with $\Delta$-excitation $\left(V_{S O}\right.$ and $W_{S O}$ collected in the appendix of ref.[20]) lead to an even smaller effect. These NN-amplitudes make up a two-body contribution to $F_{\text {so }}(\rho)$ that scales again with $1 / M$.

Finally, we show in Fig. 6 the strength function $F_{J}(\rho)$ belonging the squared spin-orbit density $\vec{J}^{2}$ in the nuclear energy density functional as a function of the nuclear density $\rho$. One observes that the inclusion of the subleading $2 \pi$-exchange strongly reduces the values of $F_{J}(\rho)$. In comparison to the (narrow) band spanned by phenomenological Skyrme forces [3, 4, 5, 6] our prediction for the strength function $F_{J}(\rho)$ is much larger in the whole density region $0<\rho<\rho_{0}$. In addition, the density dependence of $F_{J}(\rho)$ comes out markedly different, due to the long-range character of the pion-exchange interactions. For orientation, we reproduce by the dashed-dotted line in Fig. 6 the leading contribution from the $1 \pi$-exchange Fock diagram (see eq.(11)). We also note that in comparison to the calculation based on the Negele-Vautherin density-matrix expansion the magnitude of the strength function $F_{J}(\rho)$ has substantially increased (see Fig. 5 in ref.[16]).

Besides representing the non-local Fock contributions from tensor forces etc. in the energy density functional the $\vec{J}^{2}$-term leads to another interesting side effect. Namely, it gives rise to an extra spin-orbit single-particle mean-field $2 F_{J}(\rho) \vec{J}$ in addition to the "normal" one, 


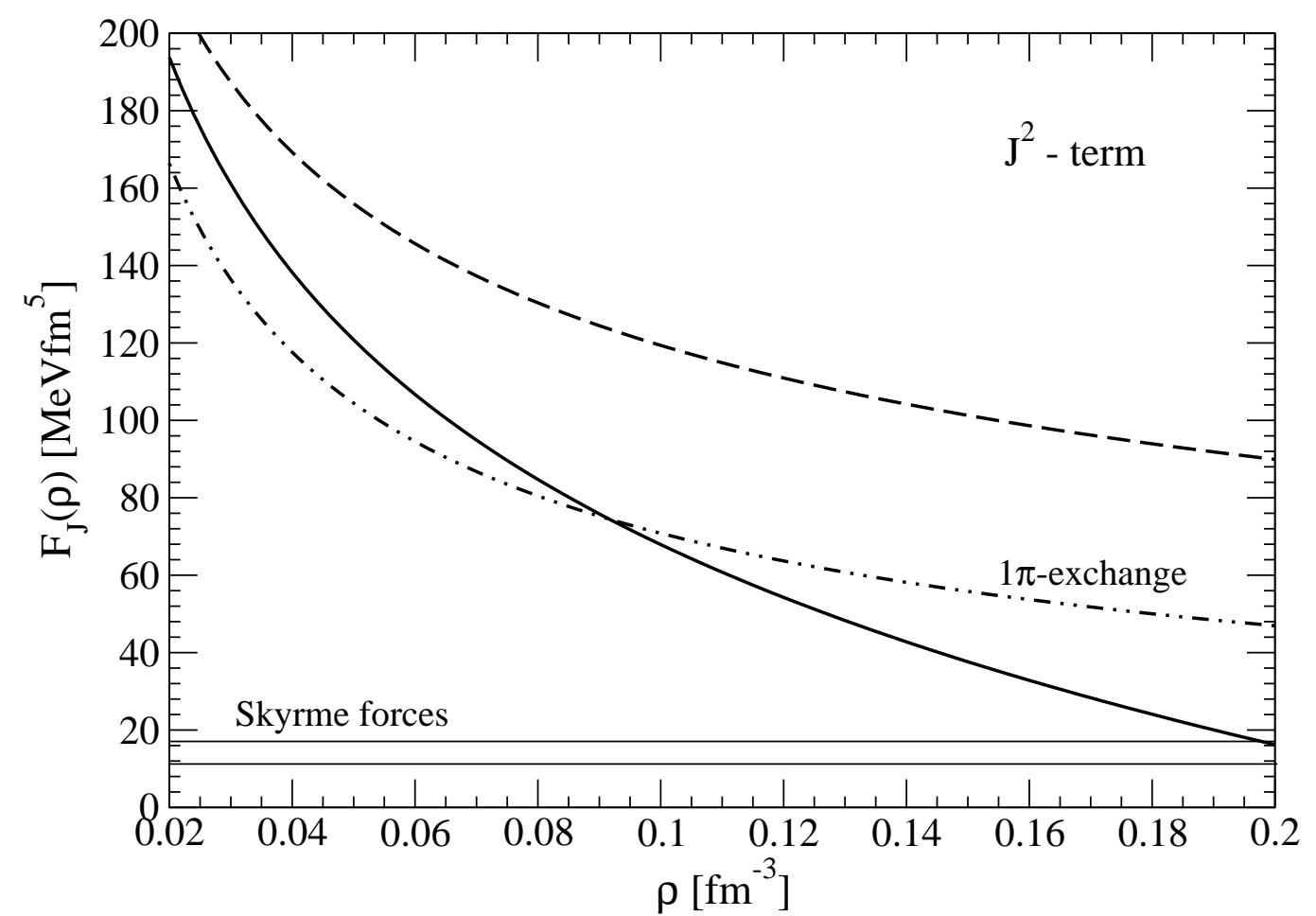

Figure 6: The strength function $F_{J}(\rho)$ accompanying the squared spin-orbit density $\vec{J}^{2}$ in the nuclear energy density functional versus the nuclear density $\rho$. Dashed line: $1 \pi$ - and iterated $1 \pi$-exchange only. Full line: $2 \pi$-exchange and three-body contributions added.

$F_{s o}(\rho) \vec{\nabla} \rho$. It would be interesting to investigate the role of this additional (nucleus-dependent) spin-orbit mean-field together with the large values and the strong density dependence of $F_{J}(\rho)$ as predicted by in-medium chiral perturbation theory.

\section{$5 \quad$ Summary and concluding remarks}

In this work we have used the recently improved density-matrix expansion of Gebremariam, Duguet and Bogner [18] to calculate the nuclear energy density functional $\mathcal{E}[\rho, \tau, \vec{J}]$ relevant for $N=Z$ even-even nuclei in the framework of in-medium chiral perturbation theory. Our calculation treats systematically the effects from $1 \pi$-exchange, iterated $1 \pi$-exchange, and irreducible $2 \pi$-exchange with intermediate $\Delta$-isobar excitations, including Pauli-blocking corrections up to three-loop order.

We find that the effective nucleon mass $M^{*}(\rho)$ entering the energy density functional becomes identical to the one of Fermi-liquid theory when employing the improved density-matrix expansion. The strength $F_{\nabla}(\rho)$ of the $(\vec{\nabla} \rho)^{2}$ surface-term as provided by the pion-exchange dynamics is in good agreement with that of phenomenological Skyrme forces in the density region $\rho_{0} / 2<\rho<\rho_{0}$.

The spin-orbit coupling strength $F_{\text {so }}(\rho)$ receives contributions from iterated $1 \pi$-exchange (of the "wrong sign") and from three-nucleon interactions mediated by $2 \pi$-exchange with virtual $\Delta$-excitation (of the "correct sign"). In the region around $\rho_{0} / 2 \simeq 0.08 \mathrm{fm}^{-3}$ where the spin-orbit interaction in nuclei gains most of its weight these two components tend to cancel, thus leaving all room for the short-range spin-orbit interaction. The empirical value $F_{s o}^{(\mathrm{emp})} \simeq 90 \mathrm{MeVfm}^{5}$ of the spin-orbit coupling strength in nuclei agrees perfectly with the one extracted from the short- 


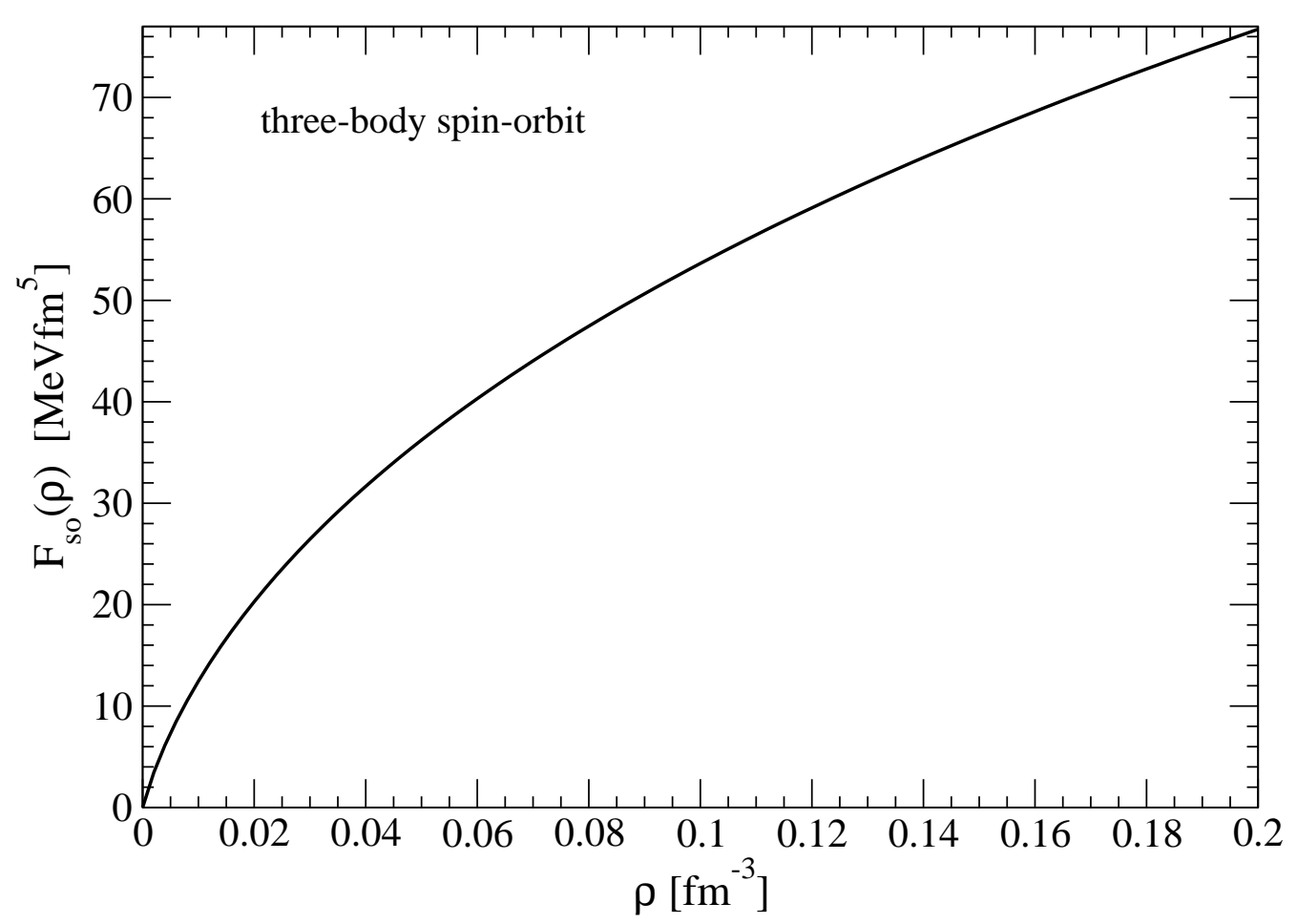

Figure 7: Three-body spin-orbit coupling strength $F_{\text {so }}(\rho)$ as a function of the nuclear density $\rho$.

range spin-orbit component of realistic NN-potentials [24]. This part of the NN-interaction drives at the same time the strong Lorentz scalar and vector mean-fields on which the whole success of the relativistic Dirac phenomenology rests.

The strength function $F_{J}(\rho)$ multiplying the squared spin-orbit density comes out much larger than from phenomenological Skyrme forces and it has a pronounced density dependence due to the long-range character of the pion-exchange interaction. The interplay between the two components of the total nuclear spin-orbit mean-field $2 F_{J}(\rho) \vec{J}+F_{s o}(\rho) \vec{\nabla} \rho$ should be further explored together with the large values and strong density dependence of $F_{J}(\rho)$ as predicted by in-medium chiral perturbation theory.

In comparison to refs. [16, 17] where the density-matrix expansion of Negele and Vautherin has been employed, we find an improved description of the nuclear energy density functional $\mathcal{E}[\rho, \tau, \vec{J}]$ on the basis of the improved density-matrix expansion [18]. In view of the fact that short-range contributions do not change (as exemplified here for the Skyrme force), a cancellation of the net two-pion exchange spin-orbit coupling strength around half nuclear matter density $\rho_{0} / 2=0.08 \mathrm{fm}^{-3}$ is more satisfactory than having this cancellation around $\rho_{0}$ as discussed in ref.[26]. In any case, the effective field theory formulation of nuclear forces provides short-range contributions to all four strength functions $F_{\tau}(\rho), F_{\nabla}(\rho), F_{\text {so }}(\rho)$ and $F_{J}(\rho)$ and these can be fine-tuned in nuclear structure calculations.

\section{Appendix: Three-body spin-orbit coupling strength}

In this appendix we present and discuss the result for the three-body spin-orbit coupling strength $F_{\text {so }}(\rho)$ one obtains from an alternative description of the $2 \pi$-exchange three-nucleon interaction. Instead of the sequential $\pi N \rightarrow \Delta \rightarrow \pi N$ transition with intermediate $\Delta$-isobar 
excitation one can employ the second order chiral $\pi \pi N N$-contact vertex [27]:

$$
\frac{i}{f_{\pi}^{2}}\left\{2 \delta_{a b}\left(c_{3} \vec{q}_{a} \cdot \vec{q}_{b}-2 c_{1} m_{\pi}^{2}\right)+c_{4} \epsilon_{a b c} \tau_{c} \vec{\sigma} \cdot\left(\vec{q}_{a} \times \vec{q}_{b}\right)\right\}
$$

to built up the $2 \pi$-exchange three-nucleon interaction. Here, $\vec{q}_{a, b}$ denote out-going pion momenta and we have already dropped the $c_{2}$ term proportional to the product of two pion energies. In the present application these (off-shell) pion energies are equal to differences of nucleon kinetic energies, thus producing a relativistic $1 / M^{2}$-correction. The pertinent in-medium diagrams are those shown in Fig. 2 with the $\Delta$-propagator shrunk to a point. Returning to the medium insertion written in eq.(5) we find from the corresponding three-body Hartree diagram (with two closed nucleon rings) the following contribution to the spin-orbit coupling strength:

$$
F_{\text {so }}(\rho)=\frac{3 g_{A}^{2} m_{\pi}}{(8 \pi)^{2} f_{\pi}^{4}}\left\{\frac{2}{u}\left(4 c_{1}-3 c_{3}\right)-4 c_{3} u+\left[\frac{4}{u}\left(c_{3}-c_{1}\right)+\frac{3 c_{3}-4 c_{1}}{2 u^{3}}\right] \ln \left(1+4 u^{2}\right)\right\},
$$

where $u=k_{f} / m_{\pi}$. It is completed by the contribution of the three-body Fock diagram (with a single closed nucleon ring) which reads:

$$
\begin{aligned}
F_{\text {so }}(\rho)= & \frac{g_{A}^{2} m_{\pi}}{\pi^{2}\left(4 f_{\pi} u\right)^{4}}\left\{3 c _ { 1 } \left[2 u-2 u^{3}+\frac{3}{2 u}-\frac{3+10 u^{2}}{4 u^{3}} \ln \left(1+4 u^{2}\right)\right.\right. \\
& \left.+\frac{3+16 u^{2}+16 u^{4}}{32 u^{5}} \ln ^{2}\left(1+4 u^{2}\right)\right]+\left(c_{3}+c_{4}\right)\left[u^{3}-\frac{16 u^{5}}{3}+\frac{7 u}{4}\right. \\
& +\frac{3}{u}+\frac{15}{16 u^{3}}+\left(2 u^{3}-\frac{3 u}{2}-\frac{13}{4 u}-\frac{39}{16 u^{3}}-\frac{15}{32 u^{5}}\right) \ln \left(1+4 u^{2}\right) \\
& \left.\left.+\frac{3}{256 u^{7}}\left(64 u^{6}+80 u^{4}+36 u^{2}+5\right) \ln ^{2}\left(1+4 u^{2}\right)\right]\right\} .
\end{aligned}
$$

Fig. 7 shows the total three-body spin-orbit coupling strength $F_{s o}(\rho)$ as a function of the nuclear density $\rho=2 k_{f}^{3} / 3 \pi^{2}$ for the choice of low-energy constants: $c_{1}=-0.81 \mathrm{GeV}^{-1}$, $c_{3}=-3.2 \mathrm{GeV}^{-1}$ and $c_{4}=5.4 \mathrm{GeV}^{-1}$. Its value at half nuclear matter density, $F_{\text {so }}\left(\rho_{0} / 2\right)=$ $47.5 \mathrm{MeVfm}^{5}$, is now about $14 \%$ larger than the analogous $\Delta$-driven three-body effects presented in section 3.7. This small increase comes mainly from the fact that the low-energy constant $-c_{3}=3.2 \mathrm{GeV}^{-1}$ is not completely saturated by its dominant $\Delta$-resonance contribution $g_{A}^{2} / 2 \Delta=2.9 \mathrm{GeV}^{-1}$.

These considerations indicate that there is some uncertainty for the density at which the cancellation between "wrong-sign" and "correct-sign" spin-orbit coupling strength actually happens. Nevertheless, the general features of such a balance remain unchanged.

\section{References}

[1] M. Bender, P.H. Heenen and P.G. Reinhard, Rev. Mod. Phys. 75 (2003) 121;

J.R. Stone and P.G. Reinhard, Prog. Part. Nucl. Phys. 58 (2007) 587.

[2] T.H.R. Skyrme, Nucl. Phys. 9 (1959) 615.

[3] M. Beiner, H. Flocard, N. Van Giai and P. Quentin, Nucl. Phys. A238 (1975) 29.

[4] J. Bartel, P. Quentin, M. Brack, C. Guet and H.B. Hakansson, Nucl. Phys. A386 (1982) 79.

[5] E. Chabanat, P. Bonche, P. Haensel, J. Meyer and R. Schaeffer, Nucl. Phys. A627 (1997) 710; A635 (1998) 231; and refs. therein. 
[6] S. Goriely, M. Samyn, M. Bender and J.M. Pearson, Phys. Rev. C68 (2003) 054325;

M. Samyn, S. Goriely, M. Bender and J.M. Pearson, Phys. Rev. C70 (2004) 044309;

N. Chamel, S. Goriely and J.M. Pearson, Nucl. Phys. A812 (2008) 72.

[7] B.D. Serot and J.D. Walecka, Int. J. Mod. Phys. E6 (1997) 515; and refs. therein.

[8] P. Ring, Lecture Notes in Physics, Vol.581, Springer-Verlag, Berlin, 2001, p. 195; and refs. therein.

[9] P. Finelli, N. Kaiser, D. Vretenar and W. Weise, Nucl. Phys. A770 (2006) 1.

[10] T. Lesinski, T. Duguet, K. Bennaceur and J. Meyer, Eur. Phys. J. A40 (2009) 121.

[11] J.E. Drut, R.J. Furnstahl and L. Platter, Prog. Part. Nucl. Phys. 64 (2010) 120; nuclth/0906.1463.

[12] S.K. Bogner, R.J. Furnstahl and L. Platter, Eur. Phys. J. A39 (2009) 219.

[13] S.K. Bogner, R.J. Furnstahl, A. Nogga and A. Schwenk, Nucl. Phys. A763 (2005) 59; nucl-th/0903.3366.

[14] R. Roth, P. Papakonstantinou, N. Paar, H. Hergert, T. Neff and H. Feldmeier, Phys. Rev. C73 (2006) 044312.

[15] J.W. Negele and D. Vautherin, Phys. Rev. C5 (1972) 1472.

[16] N. Kaiser, S. Fritsch and W. Weise, Nucl. Phys. A724 (2003) 47.

[17] S. Fritsch, N. Kaiser and W. Weise, Nucl. Phys. A750 (2005) 259.

[18] B. Gebremariam, T. Duguet and S.K. Bogner, nucl-th/0910.4979.

[19] P. Ring and P. Schuck, "The Nuclear Many-Body Problem", Springer Verlag, (1980); chapters 4 and 5 .

[20] N. Kaiser, S. Gerstendörfer and W. Weise, Nucl. Phys. A637 (1998) 395.

[21] N. Kaiser, R. Brockmann and W. Weise, Nucl. Phys. A625 (1997) 758.

[22] J. Fujita and H. Miyawawa, Prog. Theor. Phys. 17 (1957) 360; 366.

[23] N. Kaiser, Nucl. Phys. A768 (2006) 99.

[24] N. Kaiser, Phys. Rev. C70 (2004) 034307.

[25] O. Plohl and C. Fuchs, Phys. Rev. C74 (2006) 034325.

[26] N. Kaiser and W. Weise, Nucl. Phys. A804 (2008) 60.

[27] N. Kaiser, Phys. Rev. C68 (2003) 054001. 